\title{
A Theme of Smart Cities Based on IoT, Fuzzy Logic, and Quantum- Deep Learning Technique
}

\author{
Kuntal Mukherjee*1, Ranjan Kumar Mandal ${ }^{2}$
}

\begin{abstract}
Submitted: 02/12/2019 Accepted: 12/03/2020
Abstract: In the near future the human civilization would converge to urban places. Hence, tomorrows' cities would be highly dense. Thus in the future, it would be great challenges to provide efficient services to citizens. In this context, this manuscript has proposed an efficient architecture of different applications of smart cities using the Internet of Things (IoT), Fuzzy Logic. Moreover, an efficient architecture based on service-oriented architecture(SOA) has been proposed here. Furthermore, an integrated intelligent smart city services framework has been proposed here which can forecast different activities based on the Quantum Deep Learning concept. Simulated results have been shown to lay bare the effectiveness of the proposed approach.
\end{abstract}

Keywords: Internet Of Things, Smart City, Sensor Network, RFID

\section{Introduction}

It has been observed that there is a continuous migration of humans from rural to urban places. If it continues then more than sixty percent of the human population would reside at urban places by $2030[1]$. Keeping this in view, the future cities should be smart enough to manage their resources effectively and seamlessly for their huge inhabitants. Thus the smart cities would integrate the different public services, namely management of street light, transport and parking, surveillance of public areas, proper garbage management, efficient health management, efficient education system, etc for providing quality services to its inhabitants. These services for smart cities can be achieved with the help of cutting edge technology, namely the Internet of Things(IoT). IOT has opened a new world of the networked interconnection of the different things surrounding us including all computers and pervasive devices. It is actually a mesh of wireless networks of sensors that interconnect all living or nonliving things in our everyday life. It provides a vision of ubiquity in all respects-in terms of identification, sensing, computing, intelligence, etc [2][3][4]. The basic agenda of IoT is to establish an effective intelligent interaction among humans, machines and every object on Earth irrespective of time and place with low cost, in order to develop a smart city. Again, the architecture of IOT can be viewed in two angles-Internet based architecture and Thing based architecture[5]. In the former, the different services of Internet plays the central role whereas in the latter smart objects do it [6][7][8]. In the Internet based architecture of IOT, the different ubiquitous sensing devices at the sensing layer, is connected to Fog computing using the Network Layer, which comprises of Network Hardware and Network Protocols. The raw data so captured by these smart devices are processed at edge i.e. in Fog Computing Layer. This layer provides services with reduced latency and real-time computing. The processed data is

${ }^{1}$ BIT Mesra, Ranchi, Computer Science \& Eng., Ranchi, India ORCID ID: 0000-0001-7696-1754

${ }^{2}$ Ranchi University, Computer Science \& Eng., Hazaribag, India ORCID ID: 0000-0001-7784-8761

* Corresponding Author Email: kmukherjee@bitmesra.ac.in stored, analyzed and presented to end-users by the different services offered by cloud computing. Thus the raw data captured by sensing devices is processed and then transformed into information and knowledge formats using the diverse computational and storage clouds. Now, this information and knowledge is fabricated to develop a decision-making system for intelligence applications for smart cities, which is shown in Figure 1.

Application layer: It comprises of different application for smart cities namely, surveillance of public areas, smart garbage management, smart health management Smart Street Light etc.

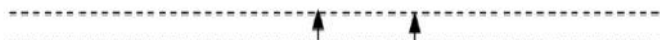

Cloud Computing Layer: Different Services of Cloud namely, Process-as-a-service, Storage-as-a-service etc. (1)

Fog Computing Layer: Processing of Data at Edge with reduced Latency and real time computing

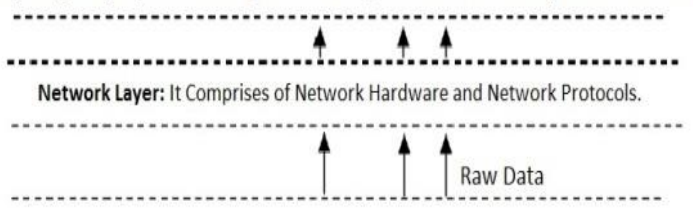

Sensing Layer: It Comprises of Sensor Network, RFID, GPS etc. to capture the Raw Data.

Figure 1. Working process of IOT

\section{Propose SOA For Smart City Based on IOT}

If Service Oriented Architecture (SOA) provides an architectural pattern in software design where different application components endow with services to other components by means of communications protocol over a network [9]. The ideology behind SOA is that the services must be independent of any vendor, product or technology and moreover services must be loosely coupled. Thus, the overall SOA technology pivots on the concept of a service, whereas a service is built as a discrete section of code. Reuse of the existing code may yield a new service and alter the interoperability of a service with other 
services and make way for a new application. For the interaction among services, the service must be aware of each other. This awareness is achieved through the use of service descriptions. A service description contains the name of the service and the data expected and returned by the service[9]. Thus SOA has come out as a boon for the corporate world by ensuring that the Information Technology system can adapt quickly, easily and economically to address rapidly changing business needs. Furthermore, it is observed that there is no defacto standard regarding the exact composition of a service-oriented architecture [9]. In this context, this endeavor has proposed IoT architecture based on SOA technology, which is shown in Figure 2.

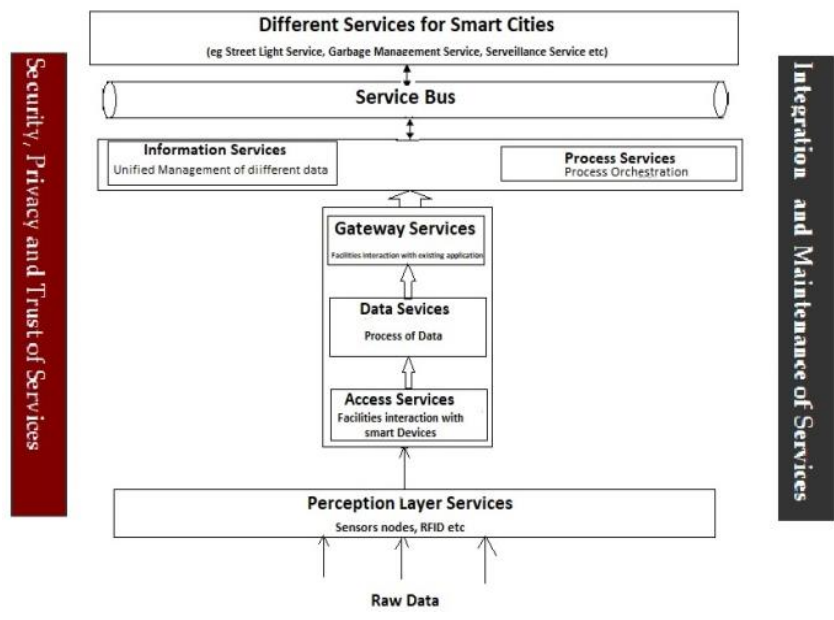

Figure 2. Proposed SOA for Smart City based on IoT

In Figure2, the role of Perception Layer Service is to acquire, collect data from the physical world using Wireless Sensor Network (WSN), Radio-Frequency Identification (RFID), Global Positioning System (GPS) etc. Furthermore, Access Services can interact with these smart devices. The processing of these raw data is done by Data Services. Moreover, Gateway Services facilities the interaction with existing applications. Now unified management of this processed data is done by Information Services. Furthermore, the process orchestration is done at Process Services. After that these different services are combined to form the smart city applications namely, Smart Street Light Service, Garbage Management Service, Surveillance Service, Health Management Service, etc. During the whole process, the integration and maintenance of these services are of the utmost need. The success of the overall smart city services depends on the security, privacy, and trust of the citizens on these services.

The organization of this endeavor consists of a brief discussion followed by the state-of-the-art of the different services of smart city thereafter the proposed architecture of different applications, namely smart street light system, proposed smart garbage management system, proposed smart surveillance system, etc. The experimental result has been shown to exhibit the effectiveness of the proposed approach and finally, conclusions with the future directions of research.

\section{Smart City Services: State-Of-Art}

The building block of the smart city is observed in the research work of [10]. Here, the authors have focused on the different issues of smart cities namely, transportation, safety, energy efficiency, etc. In literature, the different services for a smart city are observed which include intelligent street light control systems, smart garbage management systems, intelligent traffic control systems, smart parking systems, etc. In the intelligent street light control system, interesting research work has been carried out by the research scholars in [11]. Here, the authors have proposed the concept of automatic turn on-off of street lights. The street lights are turned on automatically as the vehicles or pedestrians are passing through the road. When there is no one the street lights are automatically turned off. In this way, their proposed system would not only save electrical energy but also would enhance the life span of the lighting equipment. Moreover, the intelligent street light based on a remote control system is observed in the research work of [12]. In this case, the authors have presented interesting research regarding the autonomous performance of street lights based on environment conditions namely, the presence of vehicles, people, etc. Moreover, controlling street light for smart cities based on integrated Doppler sensors is observed in [13]. When the Doppler sensors identify the increase in luminous intensity then the street light intensity increases automatically. Otherwise, they get dimmed automatically. In this way, the researchers in [13] have presented energy-efficient management for the street lights for smart cities. Apart from the street light system for the smart city, an integrated platform for the different applications for the smart city namely surveillance, intelligent traffic control system, intelligent street light, etc. is observed in the research work of [14]. Again, smart parking is also a hot topic to be addressed by smart city services. In this context, eminent research work is observed by the authors in [15]. Here the authors have described the implementation of a car park management system. The authors have made a comparative study between energy and cost while deploying a wireless sensor network and serial connection. Moreover, the authors in [16] have presented their parking sensors working on LEACH routing protocols on the Arduino platform. Again an interesting smart parking pilot program installed in Beijing, China at the year 2012 [17]. Further, garbage management is also a big issue for tomorrows' smart cities. In this regard, a lot of interesting research works are observed in the literature[18]. In fact, the garbage management for a smart city is handled using different technologies namely, Internet of things(IoT), Software Analytics, Physical Infrastructures[18]. The researcher in [19] has presented an innovative level-sensing model for the identification of the level of waste in the dustbin. Additionally, the authors in [20] have presented the Decision Support System (DSS) for waste management. The significant role of quantum computer in designing deep neural network and machine learning is observed in the research work of authors in [22]. The authors in [22] have demonstrated the significant reduction of time to train deep restricted Boltzmann machine using quantum computing as compared to classical computing. Furthermore, an interesting research regarding the use of quantum neurons in quantum feed-forward neural networks is observed in [23]. Here the proposed approach allows a fast optimisation with a reduced memory requirements. Moreover, the genesis of the concepts of fuzzy sets and its use is observed in [24]. Further, a detail discussion about the urban IOT is observed in[25]. Here, authors have presented a survey regarding technologies, protocols and architecture for an urban IoT along with the technical guidelines in detail.

\subsection{Research Gap}

But none of the research works have so far addressed an integrated environment for all smart city services. Furthermore, there is lack of research regarding an intelligent integrated Smart City Services framework. The integrated framework should take 
the decision and inform the competent authorities to take needful actions as and when required. The research regarding the development of such an intelligent framework has not been done yet.

\section{Proposed different Intelligent Services for Smart Cities}

Based upon the above discussions, in this endeavor a number of smart city applications, namely smart street light, smart garbage management system, smart vigilance system have been proposed as follows:

\section{Case-1: Proposed Smart Street Light System}

Currently, the technology used in the street light is sensor technology, more specifically Light Dependent Resistor (LDR). LDR works on the principle of light intensity. The LDR sensors test the light intensity and switch on the lights when the luminous intensity is low. But many times it is seen that the intensity of sunlight is appropriate for visibility but due to LDR sensors the lights get switched, which is simply wastage of the electrical energy. Another problem with LDR is that if dust or shadow persists on the sensor then it senses low light intensity and turns on the light. Furthermore, if the light is focused and reflected on the sensor it doesn't turn on the light in spite of the light intensity being low in the surrounding. For this, the sensor needs to be cleaned in very short intervals because the street light stands on roadways and get dirty easily by vehicle exhausts. So, the LDR needs their continuous maintenance. In contrast to this, the proposed approach in this manuscript is the automatic street light system, which is based on microcontroller. The proposed automatic street light system would accept the longitudinal and latitudinal values of a place. Based on the value of the current weather condition, time of sunset of that place the proposed system would turn the street light on accordingly. Even on a foggy day the proposed system can turn on the lights and keep them switched on as long as they are needed. The architecture of the proposed smart street light system is shown in Figure 3.

In Figure 3, the Microcontroller works on the concept of Fuzzy logic. The Microcontroller controls the relay. A relay is an electrically operated switch. Relay turn on or off the street light as per the instruction given by the microcontroller. Now at the other end, the Microcontrollers are connected with Fog Computing Layer via Bluetooth. At the Fog Computing Layer, the local processing of data is done. Furthermore, the Fog computing layer is connected with the cloud computing layer via the Internet. At the loud computing layer, weather information web service provides the current weather states of a place. At first, the longitude and latitude of a place are initialized to the microcontroller. The microcontroller passes this data to the Fog layer. The Fog layer passes this data to the Weather Information Web services present at the cloud computing layer. The Weather Information Web service accepts the longitude and latitude of a specific place and provides the weather condition, the sun set, fogging in the weather to the Fog computing layer. Now, the Fog computing layer provides this information to the microcontroller.

Now, microcontrollers based on the fuzzy rule controls the relays to turn on or off a large number of street lights. In the proposed approach, there is no need to install individual LDR for each street light. A single fuzzy-based microcontroller can control a large number of street light with very less operational expanse. Even at midnight, the microcontroller turns the street light on an alternate basis. The proposed Algorithm 1 is given below:
Algorithm 1. Smart-Street-Light:

Step 1. Input Longitude and Latitude of a place.

Step 2. Find the weather conditions of that place.

Step 3. Turn the street light on or off as per the fuzzy rule shown in Table1.

Step 4. End

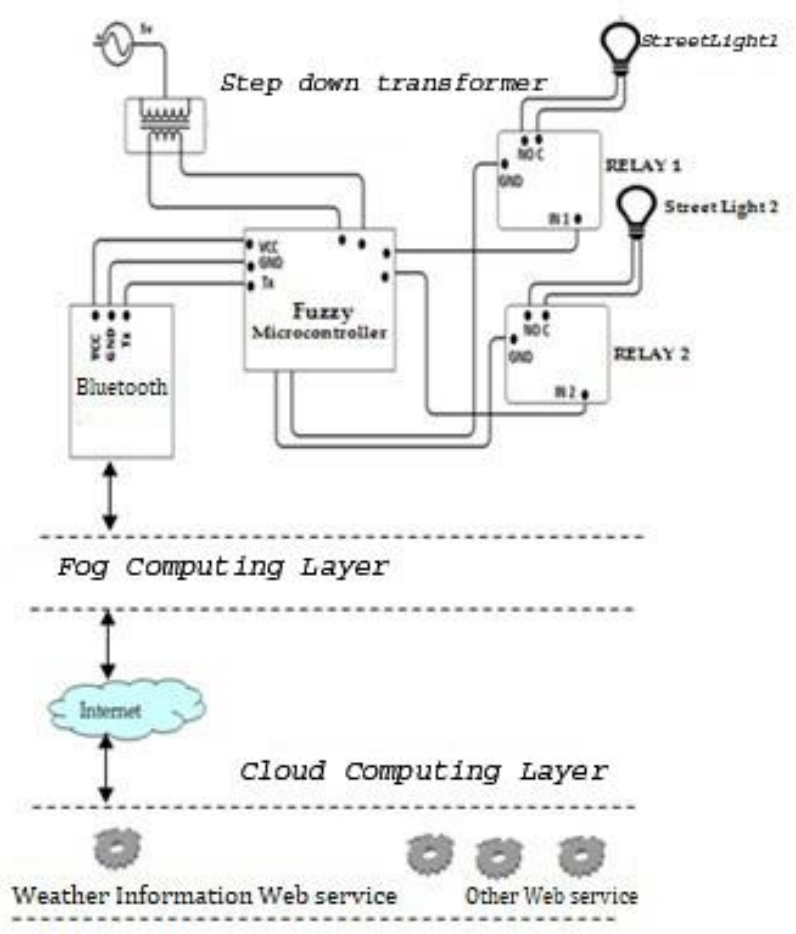

Figure 3. Proposed Architecture of Smart Street Light System

Based upon the Fuzzy rule in Table 1, the simulated result in MatLab is shown in Figures 4, 5 and in 6.

Table 1. A Fuzzy rule

\begin{tabular}{ccccc}
\hline State & Cloudy & Raining & Visibility & State of Street Light \\
\hline Day & No & No & Excellent & Off \\
Day & Yes & No & Excellent & Off \\
Day & Yes & No & Low & On \\
Day & Yes & Yes & Low & On \\
Day & No & No & Low & On \\
Day & Yes & Yes & Low & On \\
Day & Yes & Yes & Low & On \\
\hline
\end{tabular}

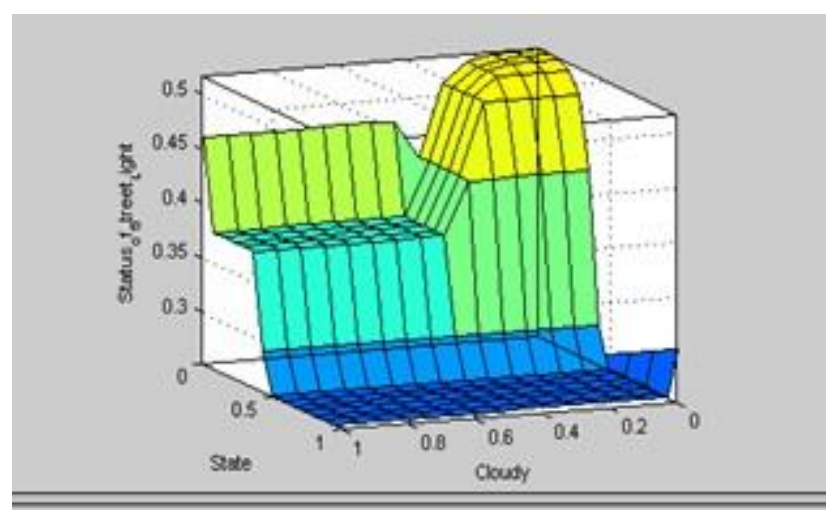

Figure 4. Simulated result of state, cloudy weather and status of street light. 


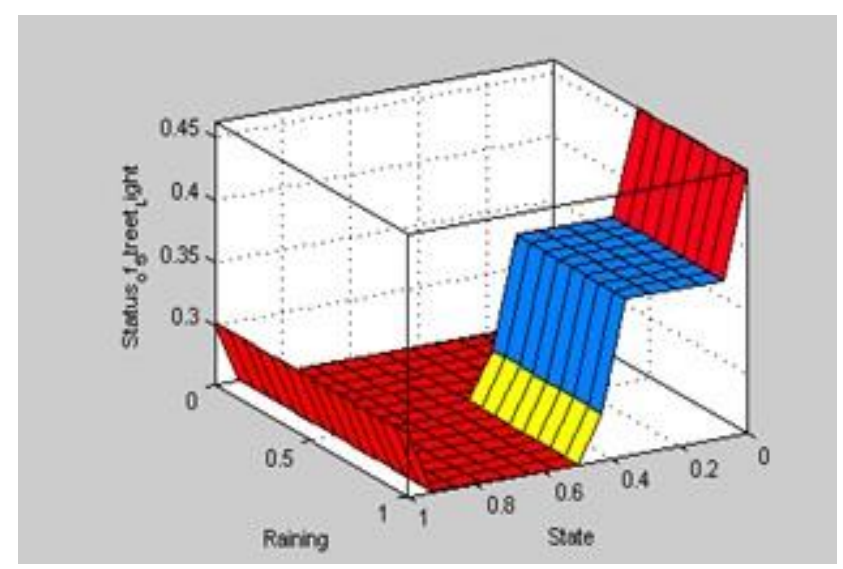

Figure 5. Simulated result of state, raining and status of street light.

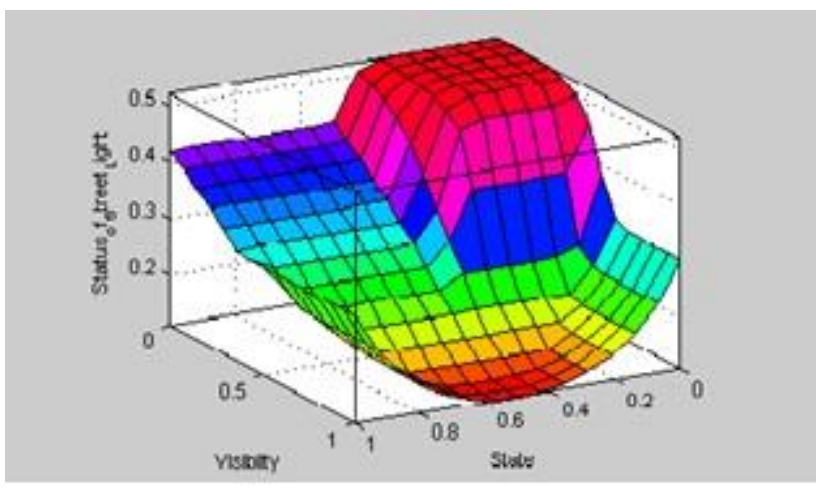

Figure 6. Simulated result of state, visibility, and status of a street light.

\section{Case-2 : Proposed Smart Garbage Management System}

In this case, another smart city application, namely a garbage management system has been proposed. Here, the dustbins are equipped with HC-SR04 ultrasonic sensors to detect the garbage level in the dustbin. If the garbage level is up to a certain level, it sends the signal to the microcontroller. The microcontroller is working on the concept of fuzzy logic and sends the signal to the Fog computing layer. Finally, the information regarding the status of the dustbin, its location is stored in the cloud storage service provided by the cloud computing layer. A mobile app, namely "Garbage Management" collects the data from the cloud storage and sends the appropriate message containing the exact location of dustbins to be emptied. The garbage collector would log in to the "Garbage Management" app and empty the dustbin on a priority basis. The Fuzzy Microcontroller generates the location, status, and priority of the dustbin to be made empty by the garbage collectors. The overall architecture of the proposed garbage management system is shown in Figure 7. The Flow Chart used for the garbage management system is shown in Figure 8. Here users would get three chances to identify the location using the app, this fact is monitored by the counter mentioned in the flow chart in Figure 8.

\section{Case-3 : Proposed Smart Surveillance System}

In this section, a smart surveillance system has been proposed. The proposed system comprised of a mesh of lamp posts (shown in Figure 9, Figure 10). The sensors for different purposes namely passive infrared sensors (measures infrared radiation from an object), acoustic sensors (can measure sound levels), active infrared sensors (can measure range and velocity of target), etc. along with the vigilance cameras(can capture image in real-time) constitute a mesh of network, where microcontroller is playing the central role. $b$
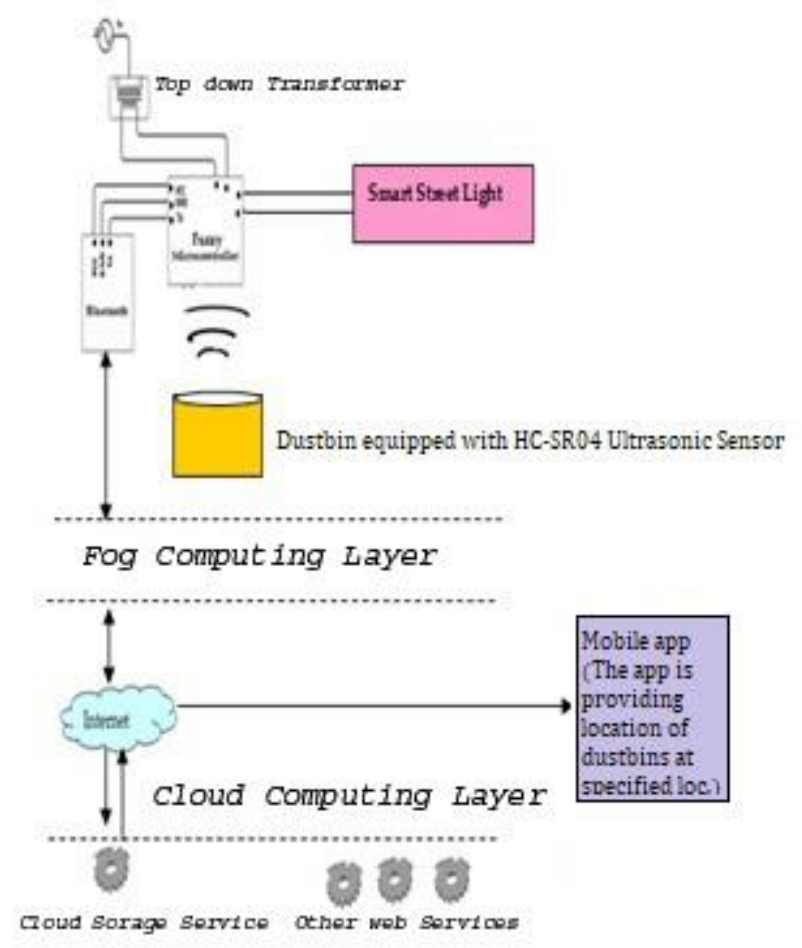

Figure 7. Proposed Architecture of Garbage Management System

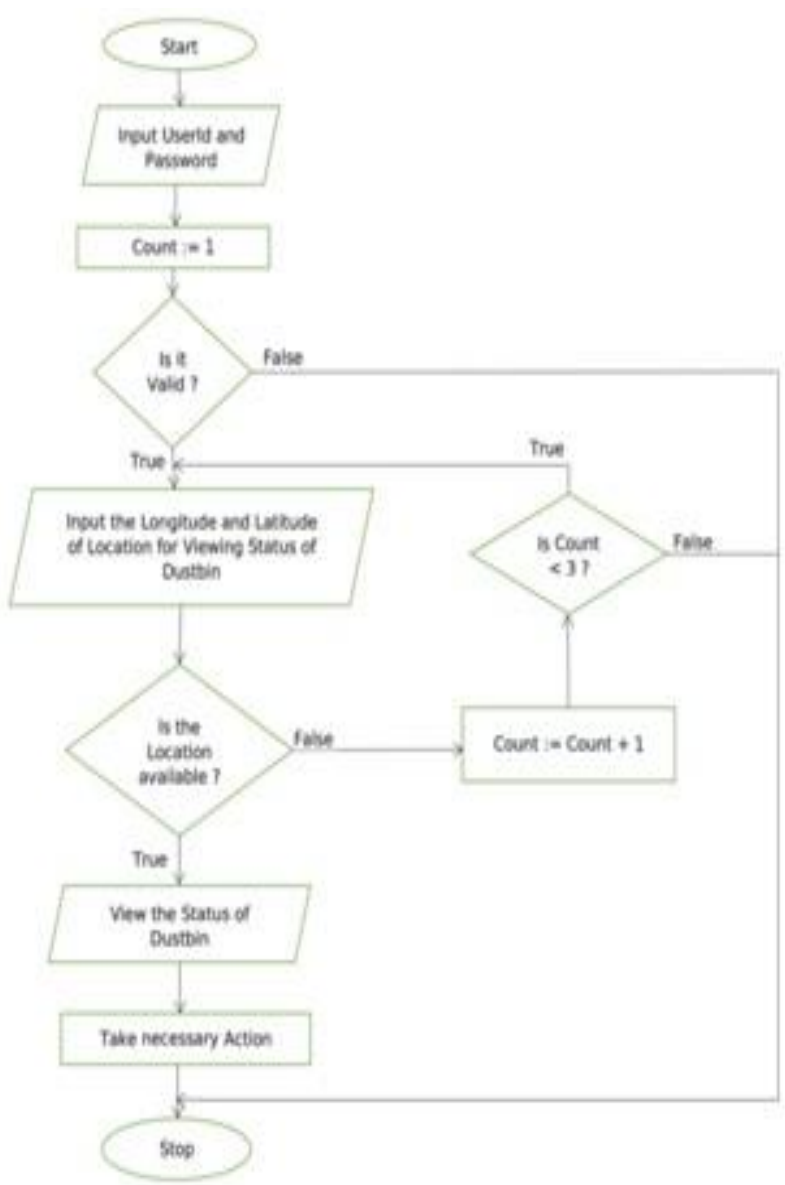

Figure 8. Flow Chart for Garbage Management System 


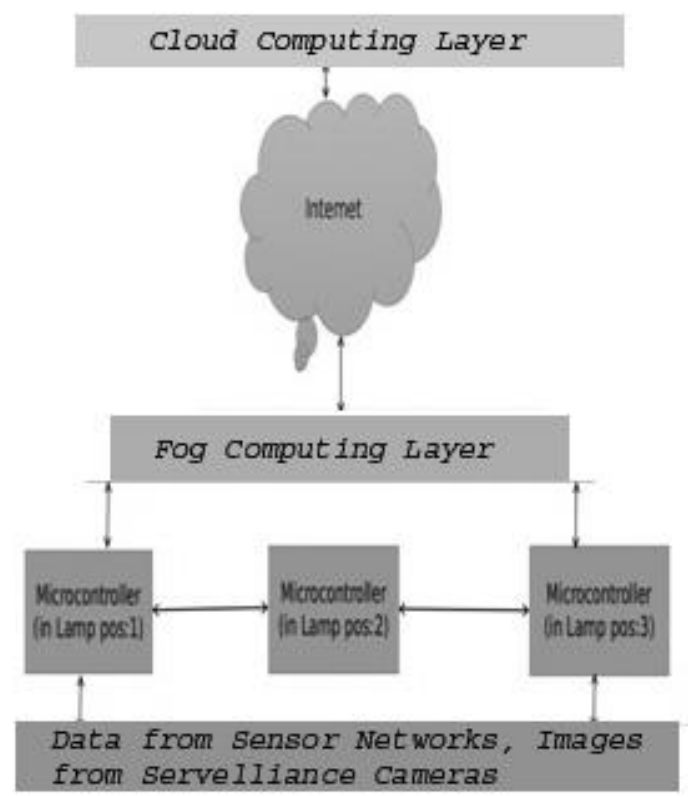

Figure 9. Mesh of Lamp Posts

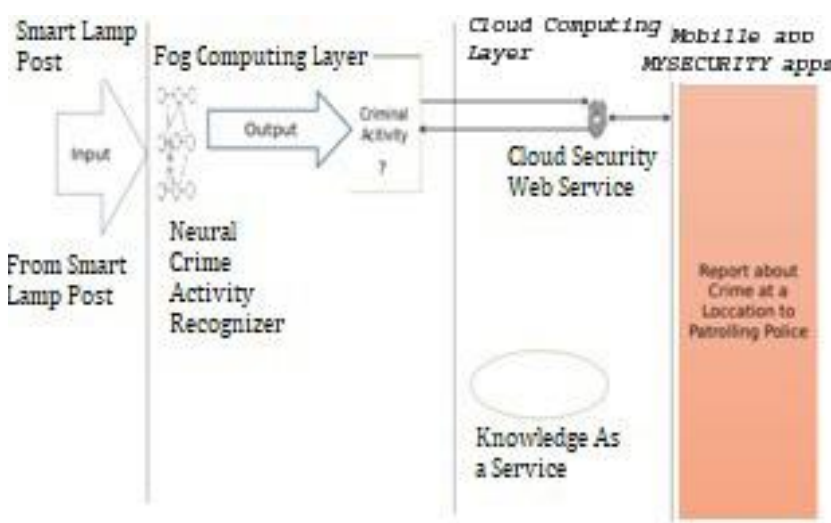

Figure 10. The architecture of the proposed Smart Surveillance System

The architecture of the proposed surveillance system is shown in Figure 10.

- A mesh of Lamp Post is made. Thus, the microcontrollers in the lamp post are connected with each other. The microcontrollers collect data from sensor networks, surveillance cameras, etc., which is shown in Figure 10.

- Now, the data so captured by the microcontrollers is sent to the Fog computing layer. Now the neural recognizer at the Fog computing layer identifies the different activities.

- Now, the neural recognizer at the Fog computing layer analyses the activity with the help of knowledge at the cloud computing layer.

- After analysis, if the quantum neural recognizer finds any criminal activity anywhere, it reports to the citizen security web service.

- The citizen security web service is providing all this information to specific Mobiles apps, namely "MYSECURITY".

- The police officers in patrolling would log in to the "MYSECURITY" apps. The "MYSECURITY" apps would continuously provide information to the patrolling police officers to control crimes.

- Thus the proposed Smart Surveillance System would help the police to maintain law and order for smart cities..

\section{Integrated Intelligent Smart City Services Framework}

The above-mentioned applications can be integrated into a smart city. The proposed intelligent Smart City Services Framework would be intelligent enough to take the decision and inform it to the competent authority to do needful. For this, three hidden layers of quantum deep learning technologies have been used here. Deep Learning is a subset of machine learning techniques. It is actually a mathematical model based on Artificial Neural Network[17]. In fact, Deep Learning comprises a number of such layers of learning representation from data. This learning representation is developed from training data and its validation is checked from test data. In this endeavor, the integrated Smart City Services framework would capture data(represented by xi, where i belongs to the set of Natural Number) through cameras, sensors, etc.

Our proposed intelligent framework, which can forecast is based on the Quantum Deep learning approach(QDL). QDL works on the principle of Quantum Mechanics (QM) defined over Hilbert Space $\left(C^{2}\right)[21]$. In Quantum computing, information is stored in qubits. A qubit is mathematically expressed by Dirac's wave function $|\psi\rangle$ as follows:

$$
|\psi\rangle=\alpha|0\rangle+\beta|1\rangle \mid \text {, Such that }|\alpha|^{2}+|\beta|^{2}=1, \alpha, \beta \in \mathbb{C}
$$

where the scalars $\alpha \in \mathrm{C}$ and $\beta \in \mathrm{C}$ are known as the base amplitudes.

The proposed intelligent framework based on quantum Feedforward Deep Neural Network for data forecasting is shown in Figure 11.

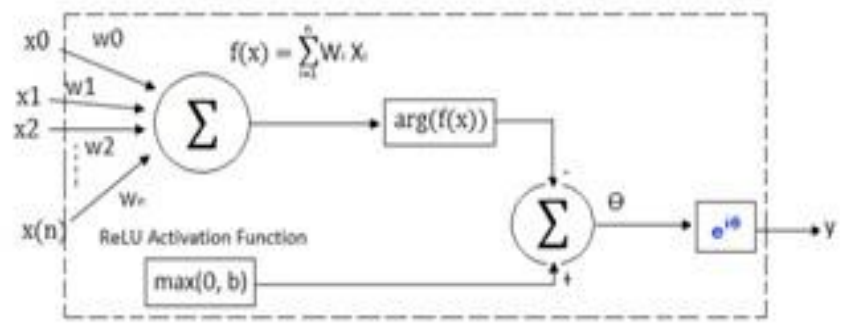

Figure 11. Quantum Feedforward Deep Neural Network

Where,

$$
\begin{aligned}
& \mathrm{f}(\mathrm{x})=\sum_{\mathbb{k}=0}^{n} W_{i} \varphi(X i) \\
& Y=x^{i[\operatorname{inax}(0, b)-\arg (f(x))]}
\end{aligned}
$$

$\mathrm{b}$ is bias and $\theta$ is the amplitude of quantum state.

The proposed algorithm for data forecasting based on QDL is shown in Figure 12. 


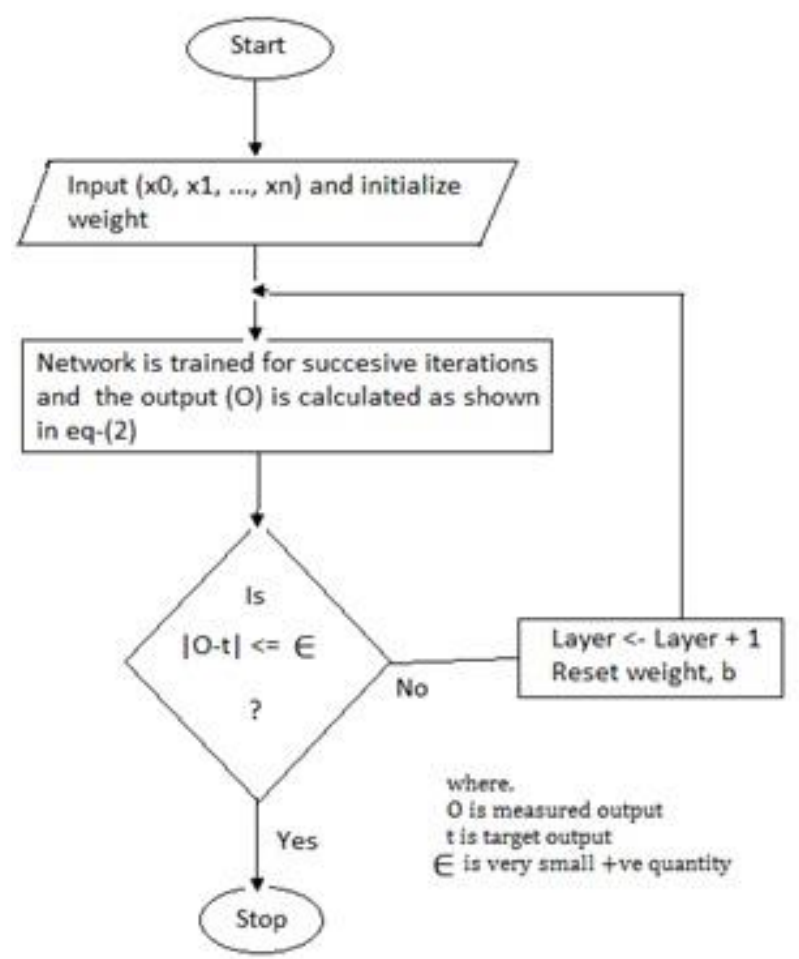

Figure 12. Proposed Flow Chart

Furthermore, the proposed architecture of integrated smart city services based on QDL Convolution Neural Network(QDL-CNN) is shown in Figure 13.

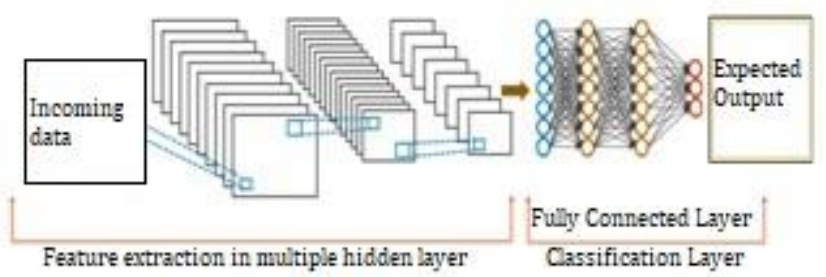

Figure 13. the architecture of the $\mathrm{CNN}$

\section{Experimental Setup and Results}

The experimental set up is made on IBMQ simulated plat- form. Here, the Quantum circuit has been made with the help of the Qiskit and python framework. Here, the Quantum Fully Connected Classification Layer is shown in Figure14.

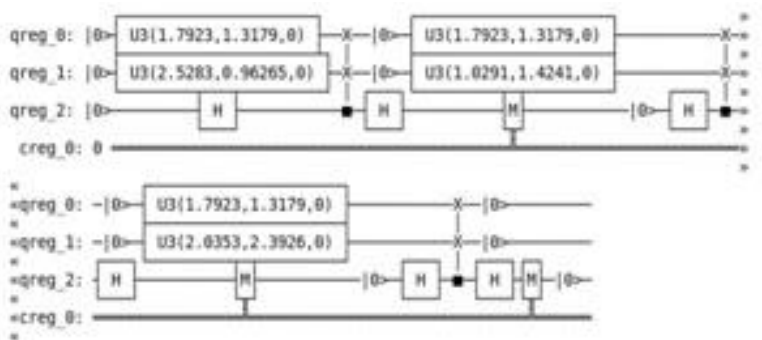

Figure 14. Quantum Fully Connected Classification Layer

Furthermore, the dataset to train the system is shown in Figure 15. The overall plot for predicted versus actual test set using data forecasting is shown in Figure 16.

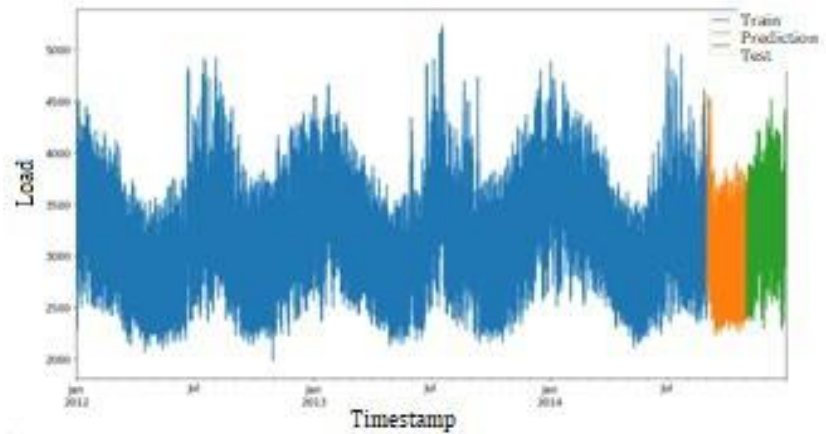

Figure 15. Dataset used to model the deep neural network

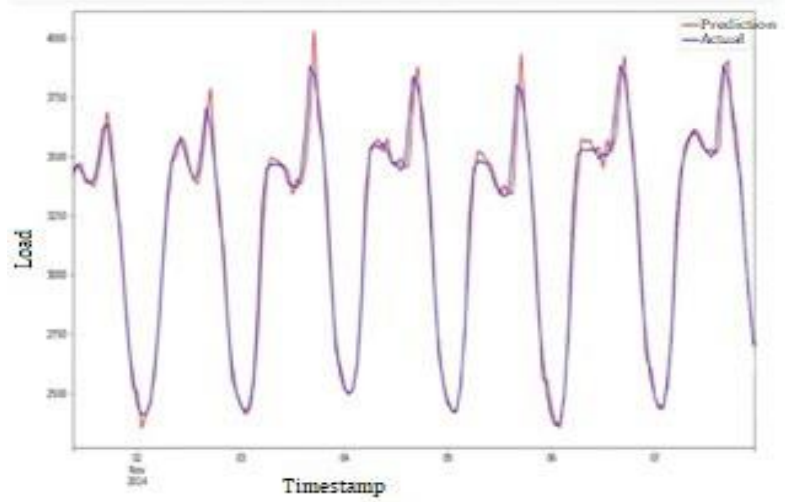

Figure 16. The overall plot for predicted versus actual test set using data forecasting

\section{Conclusions}

In this manuscript, an integrated framework of smart city services, namely smart street light system, smart garbage management system, smart vigilance system has been presented. Architectural details using a microcontroller based on fuzzy logic have been presented here. An intelligent framework that comprises all services of smart cities has been presented here based on quantum deep learning techniques. The simulated result has been shown to lay bare the effectiveness of the proposed approach. But the basic challenge of IoT enabled smart city is the energy-efficient design of different components at the sensing layer as most of the IoT devices at the sensing layer run on limited energy. Thus an energy-efficient design of IoT is the scope of future research.

\section{References}

[1] Petrolo R, Loseri V, Mitton N, Towards a smart city based on cloud of thinds, in Proceedings of the 2014 SCM International Workshop on Wireless and Mobile Technologies for Smart Cities - WiMobCity '14, New Yourk, USA: ACM Press (2014) p, 61-66 doi.10.1145/2633661.2633667.

[2] Sheng Q. Z, Zeadally S., Luo Z., Chung J. and Maamar Z., Ubiquitous RFID: Where are we?, journal of Information Systems Frontiers, Vol.12, No. 5 (November 2010) pp.485.

[3] Friedewald M., Raabe O. (May 29011) 'Ubiquitous computing: An overview of technology] impacts', Journal of Telematics and Informatics, Vol. 28, No 2, pp. 55.

[4] Pang Z., Technologies and Achitectures of the Internet-of-Things (IoT) for Health and Well-being, Doctoral Thesis in Electronic and Computer Systems KTH-Royal Institute of Technology Stockholm Sweden, (January 2013)p. 1-91.

[5] Gubbi J., Buyya R., Marusic S., and Palaniswami M., Internet of 
things (IoT): A vision, architectural elements, and future directions, Future Generation Computer Systems, Vol. 29 No. 7 (September 2013) p. 1645-1660.

[6] Lopez T. S., Ranasinghe D. C., and Harrison M., Adding sense to the internet of things: An architecture framework for smart object systems, Personal and Ubiquitous Computing, Vol. 16, No. 3(March 2012) p. 291-308.

[7] Moreno M.V., Ubeda B., Skarmeta A.F., and Amora MA., How can we tackle energy efficiency in IoT based smart buildings?, Journal of Sensors, Vol. 14 No 6 (May 2014) p. 9582-9614.

[8] Hwang Kai, Fox Geoffrey C, and Dongarra Jack J. (2012), Distributed and Cloud Computing From Parallel Processing to the Internet of Things, Morgan Kaufmann, Waltham, USA.

[9] Erl T., Service-Oriented Architecture Concepts, Technology and Design, (2013) Pearson Education Inc., India.

[10] Pavel Pribyl, Ondrej Pribyl Definition of a Smart Street as Smart City's building element, in: Proceedings of the Smart Cities Symposium Prague (SCSP), 2015.

[11]WU Yue, SHI Changhong3, ZHANG Xianghong, YANG Wiel, Design of New Intelligent Street Light Control System, $20108^{\text {th }}$ IEEE International Conference on Control and Automation Xiamen, China, June, 2010

[12]U. Hernandez-Jayo', I. Angulo', M.P. Elejoste', A. Perallos', A. Chertudi', A. Moreno', Streetlight Intelligent Remote Control System based on Wireless Communication, in: Proceedings of the $10^{\text {th }}$ International Conference on Remote Engineering and Virual Instrumentation (REV), 2013.

[13]Alexandru Lavric, Valentin Ppa, Stefan Sfichi, Street Lighting Control System Based On LargeScale WSN: A Step Towards A Smart City, in: Proceedings of the International Conference and Exposition Electrical and Power Engineering (EPE), 2014, p. $673-$ 676.

[14]Ruba A. Amarin, Steve Rhoades, Efficient Energy Solutions Enabling Smart City Deployment, in : Proceedings of the Future Technologies Conference (FTC), December, 2016.

[15] Trista Lin, Gerve Rivano, and Frederic Le Mouel, A Survey of Smart Parking Solutions, IEEE Transactions on Intelligent Transportation Systems, Volume: PP, Issue: 99(2017) p-25.

[16]D. Djenouri, E. Karbab, S. Boulkaboul, and A. Bagula, Networked wireless sensors, active RFID, and handheld devices for modern car park management: WSN, RFID, and Mob Devs for car park management, Int. J. Handheld Comput. Res., vol. 6, no. 3 (2015) $33-$ 45 .

[17] China Parking, accessed on Sep. 2016. [Online]. Available: http://www.chinaparking.org. (accessed 10.05.2017).

[18] Theodoros Anagnostopoulos, Arkady Zaslavsky, Kostas Kolomvatsos, Alexey Medvedev, Pouria Amirian, Jeremy Morley, Stathes Hadjieftymiades, Challenges and Opportunities of Waste Management in IoT enabled Smart Cities: A Survey, IEEE Transactions on Sustainable Computing, Volume PP, Issue 99(2017) $1-1$

[19]F Reverter, M. Gasulla, and R. Pallas-Areny, Capacitive level sensing for solid-waste collection, in: Proceedings of the IEEE Conference on Sensors, Vol. 1 (2003) p. 7-11.

[20]P. P. Repoussis, D. C. Paraskevopoulos, G. Zobolas, C. D. Tarantilis, and G. loannou, A web-based decision support system for waste lube oils collection and recycling, European Journal of Operational Research Vol. 195 (3), 2009, p. 676-700.

[21] N. Matsui, M. Takai, and H. Nishimura. A network model based on qubit like neuron corresponding to quantum circuit. Electronics and Communications in Japan (Part III: Fundamental Electronic Science), 83(10):67-73, 2000

[22] Nathan Wiebe, Ashish Kapoor, and Krysta M. Svore. Quantum Deep
Learning. https://arxiv.org/abs/1412.3489

[23] Kerstin Beer, Dmytro Bondarenko, Terry Farrelly, Tobias J. Osborne, Robert Salzmann, and Ramona Wolf. Efficient Learning of Deep Quantum Neural Networks. https://arxiv.org/abs/1902.10445

[24]Lotfi A. Zadeh. Fuzzy login-a personal perspective. https://doi.org/10.1016/j.fss.2015.05.009

[25] Andra Zanella, Nicola Bui, Angelo Castellani, Lorenzo Vangelista, Michele Zorzi. Internet of Things for Smart Cities. https://doi.org/10.1109/JIOT.2014.2306328 